\title{
Finite-Time Stabilization of Dynamic Nonholonomic Wheeled Mobile Robots with Parameter Uncertainties
}

\author{
Hua Chen, ${ }^{1}$ Wen Chen, ${ }^{2,3}$ Chaoli Wang, ${ }^{4}$ Dongkai Zhang, ${ }^{5}$ and Binwu Zhang ${ }^{1}$ \\ ${ }^{1}$ Mathematics and Physics Department, Hohai University, Changzhou Campus, Changzhou 213022, China \\ ${ }^{2}$ College of Mechanics and Materials, Hohai University, Nanjing 210098, China \\ ${ }^{3}$ State Key Laboratory of Hydrology-Water Resources and Hydraulic Engineering, Hohai University, Nanjing 210098, China \\ ${ }^{4}$ Department of Control Science and Engineering, University of Shanghai for Science and Technology, Shanghai 200093, China \\ ${ }^{5}$ Department of Mathematics, Shijiazhuang University, Shijiazhuang 050035, China
}

Correspondence should be addressed to Hua Chen; chenhua112@163.com

Received 20 September 2013; Accepted 13 November 2013

Academic Editor: Jin Liang

Copyright (C) 2013 Hua Chen et al. This is an open access article distributed under the Creative Commons Attribution License, which permits unrestricted use, distribution, and reproduction in any medium, provided the original work is properly cited.

\begin{abstract}
The finite-time stabilization problem of dynamic nonholonomic wheeled mobile robots with parameter uncertainties is considered for the first time. By the equivalent coordinate transformation of states, an uncertain 5-order chained form system can be obtained, based on which a discontinuous switching controller is proposed such that all the states of the robots can be stabilized to the origin equilibrium point within any given settling time. The systematic strategy combines the theory of finite-time stability with a new switching control design method. Finally, the simulation result illustrates the effectiveness of the proposed controller.
\end{abstract}

\section{Introduction}

Stabilization problem of nonholonomic systems is theoretically challenging and practically interesting. As pointed out in [1], although every nonholonomic system is controllable, it cannot be stabilized to a point with pure smooth (or even continuous) state feedback law. In order to overcome the difficulty of Brocket's condition [1], a variety of sophisticated feedback stabilization methods have been proposed which mainly include continuous time-varying feedback control laws [2-4], discontinuous feedback control laws [5-8], and hybrid feedback control laws [9].

A common characteristic of these designs of controllers above is based on kinematic model, where only a kinematic model is considered and the velocities are taken as control inputs. But in fact, for some mechanical systems with nonholonomic constraints, it is more realistic to formulate the control problems at dynamic levels, where the torque and force are chosen as new inputs. Some results can be found in recent papers, for example, the dynamic tracking control of wheeled mobile robots in the presence of both actuator saturations and external disturbances is considered in [10], where a computationally tractable moving horizon $\mathscr{H}_{\infty}$ tracking scheme is presented. In $[11,12]$, the saturated stabilization and tracking control are discussed for simple dynamic nonholonomic mobile robot. For uncertain dynamic nonholonomic systems, Ma and Tso [13] have given a robust control law for the exponential regulation of an uncertain dynamic nonholonomic wheeled mobile robot, in which the authors improved the convergence speed of regulating the state to a desired set point for the first time.

In order to drive a system to the equilibrium point with a fast convergence rate, finite-time stability theory has become a studying focus recently, for example, finitetime stabilization problems have been studied mostly in the contexts of optimality, controllability, and deadbeat control for several decades [14-16]. Compared to the asymptotic stabilization, the finite-time stabilization, which renders the trajectories of the closed-loop systems convergent to the origin in a finite time, has many advantages such as fast response, high tracking precision, and disturbance rejection properties.

For the nonholonomic systems, a few researchers have got some excellent results in finite-time control field. In [17], 
the relay switching technique and the terminal sliding mode control scheme with finite-time convergence are used for the design of the controller to address the tracking control of the nonholonomic systems with extended chained form. For a class of uncertain nonholonomic chained form systems, Hong et al. [18] have designed a nonsmooth state feedback law such that the controlled chained form system is both Lyapunov stable and finite-time convergent within any given settling time. And the finite-time tracking control for single mobile robots or multiple nonholonomic mobile robots is considered in [19-21]. The previous developed controller for nonholonomic systems can be divided into two categories: one is for the finite-time stabilization problem of chained form systems and the other is for the tracking control problem of mobile robots. However, to the best of our knowledge, there exist no results to deal with the robust finite-time stabilization of uncertain dynamic nonholonomic mobile robots.

This paper considers the stabilization problem of dynamic nonholonomic mobile robots with uncertain parameters in a finite time. The main results and contributions can be summarized as the following two respects.

(a) An uncertain 5-order chained form system can be obtained under the equivalent coordinate transformation of states, which means the finite-time stabilization of the chained form system is equivalent to the finite-time stabilization of the original dynamic robot system.

(b) Applying the theory of finite-time stability and the switching control method, we design a discontinuous robust controller to make the states of the chained form system converge to the equilibrium point in a finite time.

The structure of the paper is as follows: Section 2 gives a formalization of the problem considered in the paper. Section 3 states our main results. Section 4 provides an illustrative numerical example and the corresponding simulation results of the proposed methodology. Finally, a conclusion is shown in Section 5.

\section{Problem Statement}

A class of nonholonomic wheeled mobile robots are shown in Figure 1, the two fixed rear wheels of the robot are controlled independently by motors, and a front castor wheel prevents the robot from tipping over as it moves on a plane. Assuming that the geometric center point and the mass center point of the robot are the same and that the radiuses $r$ are identical for all the rear wheels, $2 R$ is the length of the fixed two rear wheels, where $r$ and $R$ are known positive constants. Its kinematic and dynamics model can be described by the following differential equations [13]:

$$
\begin{gathered}
\dot{x}=v \cos \theta, \quad \dot{y}=v \sin \theta, \quad \dot{\theta}=\omega, \\
m \dot{v}=\frac{\tau_{1}+\tau_{2}}{r}, \quad I \dot{\omega}=\frac{R}{r}\left(\tau_{1}-\tau_{2}\right),
\end{gathered}
$$

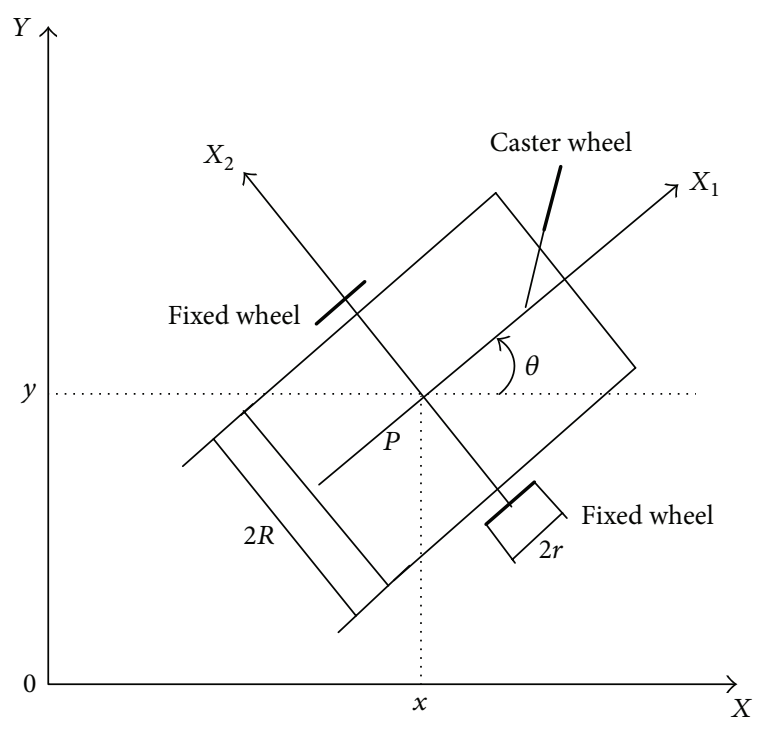

FIgURE 1: Nonholonomic wheeled mobile robot.

where $x, y$ are the position of the mass center $P$ of the robot moving in the plane and $m, I$ are the mass and inertia of mobile robots; respectively, $v$ is the forward velocity, $\omega$ is the steering velocity and $\theta$ denotes its heading angle from the horizontal axis, and $\tau_{1}, \tau_{2}$ are driving torques on the right and left rear wheels.

The geometric and inertia parameters $(r, R, m, I)$ are all assumed to be unknown positive constants, but are bounded by some known positive constants $\left(r_{m}, r_{M}, R_{m}, R_{M}, m_{m}, m_{M}\right.$, $\left.I_{m}, I_{M}\right)$, that is,

$$
\begin{array}{lc}
0<r_{m} \leq r \leq r_{M}, & 0<R_{m} \leq R \leq R_{M}, \\
0<m_{m} \leq m \leq m_{M}, & 0<I_{m} \leq I \leq I_{M} .
\end{array}
$$

One of the equilibrium states of systems $(1)$ is $(x, y, \theta, v, \omega)=$ $(0,0,0,0,0)$.

The control objective is to design a discontinuous state feedback law $\tau_{1}(x, y, \theta, v, \omega), \tau_{2}(x, y, \theta, v, \omega)$ such that the state trajectory $(x, y, \theta, v, \omega)$ of dynamic nonholonomic mobile robot system (1) starting from an arbitrary initial state $(x(0), y(0), \theta(0), v(0), \omega(0))$ converges to the origin equilibrium point in a finite time with the unknown parameters $(r, R, m, I)$ satisfying (2).

As pointed out in [11], take an orthogonal coordinate transformation

$$
\left(\begin{array}{l}
x_{1} \\
x_{2} \\
x_{3} \\
x_{4} \\
x_{5}
\end{array}\right)=\left(\begin{array}{ccccc}
0 & 0 & 1 & 0 & 0 \\
\cos \theta & \sin \theta & 0 & 0 & 0 \\
\sin \theta & -\cos \theta & 0 & 0 & 0 \\
0 & 0 & 0 & 0 & 1 \\
0 & 0 & 0 & 1 & 0
\end{array}\right)\left(\begin{array}{l}
x \\
y \\
\theta \\
v \\
\omega
\end{array}\right)
$$


Then system (1) can be converted to the following equation:

$$
\begin{aligned}
& \dot{x}_{1}=x_{4}, \\
& \dot{x}_{2}=x_{5}-x_{3} x_{4}, \\
& \dot{x}_{3}=x_{2} x_{4}, \\
& \dot{x}_{4}=c_{1} u_{1}, \\
& \dot{x}_{5}=c_{2} u_{2},
\end{aligned}
$$

where $u_{1}=\tau_{1}-\tau_{2}, u_{2}=\tau_{1}+\tau_{2}$ are new control inputs and $c_{1}=R / r I, c_{2}=1 / \mathrm{rm}$ are new unknown parameters with their bounds derived from (2) as follows:

$$
\begin{aligned}
& 0<c_{1 m}=\frac{R_{m}}{r_{M} I_{M}} \leq c_{1} \leq \frac{R_{M}}{r_{m} I_{m}}=c_{1 M}, \\
& 0<c_{2 m}=\frac{1}{r_{M} m_{M}} \leq c_{2} \leq \frac{1}{r_{m} m_{m}}=c_{2 M} .
\end{aligned}
$$

Because the coordinate transformation (3) is globally invertible and does not change the origin, it is obvious that the equilibrium point $\left(x_{1}, x_{2}, x_{3}, x_{4}, x_{5}\right)=(0,0,0,0,0)$ is finitetime stable for its closed-loop system of (4) it is means that $(x, y, \theta, v, \omega)=(0,0,0,0,0)$ is also a finite time stable equilibrium point for the corresponding closed-loop system of (1).

Hence, the control task is to design a discontinuous finitetime stabilizing controller for system (4) with the unknown parameters (5). Here, it should be noted that Hong et al. have designed a switching control strategy to discuss the finitetime stabilization of uncertain chained form systems in [18], however, it is invalid to control the dynamic chained system (4); thus, a new improved discontinuous design method is required.

The following definition and lemmas are needed for our controller design later.

Definition 1 (see [14-16]). Consider a time-invariant system in the form of

$$
\dot{\xi}=f(\xi), \quad f(0)=0, \xi \in R^{n},
$$

where $f: \widehat{U}_{0} \rightarrow R^{n}$ is continuous on an open neighborhood $\widehat{U}_{0}$ of the origin. The equilibrium $\xi=0$ of the system is (locally) finite-time stable if (i) it is asymptotically stable, in $\widehat{U}$, an open neighborhood of the origin, with $\widehat{U} \subseteq \widehat{U}_{0}$; (ii) it is finite-time convergent in $\widehat{U}$, that is, for any initial condition $\xi_{0} \in \widehat{U} \backslash\{0\}$, there is a settling time $T>0$ such that every solution $\xi\left(t, \xi_{0}\right)$ of system (6) is defined with $\xi\left(t, \xi_{0}\right) \in \widehat{U} \backslash\{0\}$ for $t \in[0, T)$ and satisfies $\lim _{t \rightarrow T} \xi\left(t, \xi_{0}\right)=0$, and $\xi\left(t, \xi_{0}\right) \equiv 0$ if $t \geq T$. Moreover, if $\widehat{U}=R^{n}$, the origin $\xi=0$ is globally finite-time stable.

Lemma 2 (see $[15,18])$. Suppose there exist a $C^{1}$ positivedefinite and proper function $\bar{V}_{1}: R^{n} \rightarrow R$, real numbers $\bar{k}>0, \bar{\beta} \in(0,1)$, such that $\dot{\bar{V}}_{1}+\bar{k} \bar{V}_{1}^{\bar{\beta}}$ is negative semidefinite. Then, the origin is a globally finite-time stable equilibrium point of system (6).
Lemma 3 (see [18]). For the uncertain time-varying chained form system:

$$
\begin{aligned}
& \dot{z}_{1}=q_{1} z_{2} u_{0}, \\
& \vdots \\
& \dot{z}_{n-1}=q_{n-1} z_{n} u_{0}, \\
& \dot{z}_{n}=q_{n} u+\psi_{n}(z),
\end{aligned}
$$

where $z=\left[z_{1}, z_{2}, \ldots, z_{n}\right]^{T} \in R^{n}, u \in R$ are the state and control input, respectively; $q_{i}>0(i=1,2, \ldots, n)$ are uncertain parameters located in known intervals, that is,

$$
0<q_{i} \in\left[q_{i}^{\min }, q_{i}^{\max }\right], \quad i=1,2, \ldots, n
$$

$\psi_{n}(z)$ is an uncertain function satisfying

$$
\left|\psi_{n}(z)\right| \leq M \sum_{i=1}^{n}\left|z_{i}\right|, \quad M>0,
$$

and $u_{0}(t)$ satisfies $0<\gamma \leq\left|u_{0}(t)\right| \leq \widehat{\gamma}$.

Let $r_{i}>0, \beta_{i-1}>0, i=1,2, \ldots, n$ and $k=p / q-1<0$ (with $q>0$ and $p>0$ odd integers) be real numbers satisfying

$$
\begin{gathered}
r_{1}=1, \ldots, r_{i}=r_{i-1}+k, \quad r_{i}>-k>0, i=1,2, \ldots, n, \\
\beta_{0}=r_{2}, \quad\left(\beta_{i}+1\right) r_{i+1}=\left(\beta_{i-1}+1\right) r_{i}>0, \\
i=1,2, \ldots, n-1 .
\end{gathered}
$$

Then, finite-time stabilizing control law of (7) can be constructed in the form of

$$
u=v_{n}=-\gamma l_{n} w_{n}^{\left(r_{n}+k\right) /\left(r_{n} \beta_{n}-1\right)}-\frac{M}{q_{n}^{\min }}\left(\sum_{i=1}^{n}\left|z_{i}\right|\right) \operatorname{sgn}\left(w_{n}\right),
$$

where $w_{n}$ is defined as follows:

$$
\begin{gathered}
w_{1}=z_{1}^{1+k}, \quad w_{i}\left(z_{1}, \ldots, z_{i}\right)=z_{i}^{\beta_{i-1}}-v_{i-1}^{\beta_{i-1}}, \\
v_{i-1}=-l_{i-1} w_{i-1}^{\left(r_{i-1}+k\right) /\left(r_{i-1} \beta_{i-2}\right)} \operatorname{sgn}\left(u_{0}\right), \quad i=2, \ldots, n,
\end{gathered}
$$

where $\operatorname{sgn}(\cdot)$ denotes the sign function and $l_{i}>0, i=1, \ldots, n$ are suitable constants.

Proof. See [18] for details.

\section{Main Results}

In this section, the main results will be presented. Firstly, we will state the basic idea to design a finite-time switching controller for system (4).

Note that system (4) can be decoupled into two subsystems, one of which is $\left\{x_{1}, x_{4}\right\}$-subsystem

$$
\begin{gathered}
\dot{x}_{1}=x_{4}, \\
\dot{x}_{4}=c_{1} u_{1},
\end{gathered}
$$


and the other describes the rest of (4), that is, $\left\{x_{3}, x_{2}, x_{5}\right\}$ subsystem

$$
\begin{gathered}
\dot{x}_{3}=x_{2} x_{4}, \\
\dot{x}_{2}=x_{5}-x_{3} x_{4}, \\
\dot{x}_{5}=c_{2} u_{2} .
\end{gathered}
$$

By designing $u_{1}$, the state $x_{4}$ of (13) can be driven to any predetermined point in a finite time, based on which $\left(x_{3}, x_{2}, x_{5}\right)$ of $(14)$ can be stabilized to $(0,0,0)$ by designing the finite-time controller $u_{2}$, and the last step is to redesign $u_{1}$ such that $\left(x_{1}, x_{4}\right)$ can be driven to $(0,0)$ in a finite time.

The following are the switching controller design and the corresponding proofs of Theorems 4 and 6.

Theorem 4. Given $c_{0}>0$, for system (4), take the following switching control law.

Step 1. Let $u_{1}=-k_{1} \operatorname{sgn}\left(x_{4}-c_{0}\right)\left|x_{4}-c_{0}\right|^{\alpha_{1}}, u_{2}=0$, where $k_{1}>0, \alpha_{1} \in(0,1)$. Until $x_{4}=c_{0}$, then go to Step 2 .

Step 2. Let

$$
\begin{gathered}
u_{1}=-k_{1} \operatorname{sgn}\left(x_{4}-c_{0}\right)\left|x_{4}-c_{0}\right|^{\alpha_{1}}, \\
u_{2}=\widetilde{v}_{3}=-l_{3} \widetilde{w}_{3}^{\left(r_{3}+k\right) /\left(r_{3} \beta_{3}-1\right)}-\frac{c_{0}}{c_{2 m}}\left(\sum_{i=1}^{3}\left|y_{i}\right|\right) \operatorname{sgn}\left(\widetilde{w}_{3}\right),
\end{gathered}
$$

where

$$
\begin{gathered}
\widetilde{w}_{1}=y_{1}^{1+k}, \quad \widetilde{w}_{i}\left(y_{1}, \ldots, y_{i}\right)=y_{i}^{\beta_{i-1}}-\widetilde{v}_{i-1}^{\beta_{i-1}}, \\
\widetilde{v}_{i-1}=-l_{i-1} \widetilde{w}_{i-1}^{\left(r_{i-1}+k\right) /\left(r_{i-1} \beta_{i-2}\right)}, \quad i=2,3, \\
y_{1}=x_{3}, \quad y_{2}=c_{0} x_{2}, \quad y_{3}=c_{0} x_{5}-c_{0}^{2} x_{3} .
\end{gathered}
$$

Unitll $y_{i}=0, i=1,2,3$, then go to Step 3 .

Step 3. Let $u_{2}=0$,

$$
u_{1}=\bar{v}_{2}=-l_{2} \bar{w}_{2}^{\left(r_{2}+k\right) /\left(r_{2} \beta_{2}-1\right)}
$$

where

$$
\begin{gathered}
\bar{w}_{1}=x_{1}^{1+k}, \quad \bar{w}_{2}\left(x_{1}, x_{4}\right)=x_{4}^{\beta_{1}}-\bar{v}_{1}^{\beta_{1}}, \\
\bar{v}_{1}=-l_{1} \bar{w}_{1}^{\left(r_{1}+k\right) /\left(r_{1} \beta_{0}\right)} .
\end{gathered}
$$

Until $x_{1}=x_{4}=0$, stop.

Then system (4) can be stabilized to the origin equilibrium point in a finite time by the switching controller Step 1-Step 3.

Proof. In the first step, let $\eta=x_{4}-c_{0}, V_{1}=(1 / 2) \eta^{2}$, then

$$
\begin{gathered}
\dot{\eta}=\dot{x}_{4}=c_{1} u_{1}=-c_{1} k_{1} \operatorname{sgn}(\eta)|\eta|^{\alpha_{1}}, \\
\dot{V}_{1}=\eta \dot{\eta}=-c_{1} k_{1}|\eta|^{1+\alpha_{1}}=-c_{1} k_{1}\left(2 V_{1}\right)^{\left(1+\alpha_{1}\right) / 2},
\end{gathered}
$$

which means that $\dot{V}_{1}+c_{1} k_{1}\left(2 V_{1}\right)^{\left(1+\alpha_{1}\right) / 2} \leq 0$; according to the conclusion of Lemma 2 , there exists a finite time $T_{1}>0$ such that $\eta=0$ for all $t \geq T_{1}$, that is, $x_{4}=c_{0}$ after this time.
In Step 2, for the subsystem (14)

$$
\begin{gathered}
\dot{x}_{3}=x_{2} c_{0}, \\
\dot{x}_{2}=x_{5}-x_{3} c_{0}, \\
\dot{x}_{5}=c_{2} u_{2} .
\end{gathered}
$$

Let

$$
\left(\begin{array}{l}
y_{1} \\
y_{2} \\
y_{3}
\end{array}\right)=\left(\begin{array}{ccc}
1 & 0 & 0 \\
0 & c_{0} & 0 \\
-c_{0}^{2} & 0 & 0
\end{array}\right)\left(\begin{array}{l}
x_{3} \\
x_{2} \\
x_{5}
\end{array}\right) .
$$

One has

$$
\begin{aligned}
& \dot{y}_{1}=y_{2}, \\
& \dot{y}_{2}=y_{3}, \\
& \dot{y}_{3}=c_{0} c_{2} u_{2}-c_{0}^{2} y_{2} .
\end{aligned}
$$

Because $\operatorname{det}(A)=c_{0}^{2}>0$, where

$$
A=\left(\begin{array}{ccc}
1 & 0 & 0 \\
0 & c_{0} & 0 \\
-c_{0}^{2} & 0 & c_{0}
\end{array}\right),
$$

the finite-time stabilization problems of (20) and (22) are equivalent.

Set $z_{i}=y_{i}(i=1,2,3), q_{1}=q_{2}=u_{0}=1, q_{3}=c_{0} c_{2}$, $\psi_{3}(z)=-c_{0}^{2} z_{2}$, and from Lemma 3, system (20) can be stabilized to zero by $u_{2}$ in Step 2 after some finite time $T_{2}$; that is, $x_{3}=x_{2}=x_{5}=0$ for all $t \geq T_{2}$.

Similarly, consider subsystem (13) again in the last step

$$
\begin{gathered}
\dot{x}_{1}=x_{4}, \\
\dot{x}_{4}=c_{1} u_{1} .
\end{gathered}
$$

Set $z_{1}=x_{1}, z_{2}=x_{4}, q_{1}=u_{0}=1, q_{2}=c_{1}, \psi_{2}(z)=0$, by using Lemma 3 , it is clear that there exists a finite time $T_{3}$ such that system (13) can also be stabilized to zero after $t \geq T_{3}$.

This completes the proof of the theorem.

Remark 5. Then control objective can be completed in each step within a finite time, and thus system (4) can be stabilized to zero in a finite time.

On the other hand, from (3), we have the following:

$$
\begin{aligned}
x=x_{2} \cos \theta+x_{3} \sin \theta & =x_{2} \cos x_{1}+x_{3} \sin x_{1}, \\
y=x_{2} \sin \theta-x_{3} \cos \theta & =x_{2} \sin x_{1}-x_{3} \cos x_{1}, \\
\theta & =x_{1}, \\
v & =x_{5}, \\
\omega & =x_{4} .
\end{aligned}
$$

Therefore, the finite-time switching controller for the original robot system (1) can be stated as follows. 
Theorem 6. Given $c_{0}>0$, for system (1), take the following switching control law.

Step $1^{\prime}$. Let

$$
\begin{aligned}
& \tau_{1}=-\frac{1}{2} k_{1} \operatorname{sgn}\left(\omega-c_{0}\right)\left|\omega-c_{0}\right|^{\alpha_{1}}, \\
& \tau_{2}=\frac{1}{2} k_{1} \operatorname{sgn}\left(\omega-c_{0}\right)\left|\omega-c_{0}\right|^{\alpha_{1}},
\end{aligned}
$$

where $k_{1}>0, \alpha_{1} \in(0,1)$. Until $\omega=c_{0}$, then go to Step $2^{\prime}$.

Step $2^{\prime}$. Let

$$
\begin{gathered}
\tau_{1}=-\frac{1}{2}\left(k_{1} \operatorname{sgn}\left(\omega-c_{0}\right)\left|\omega-c_{0}\right|^{\alpha_{1}}\right. \\
\left.+l_{3} \widetilde{w}_{3}^{\left(r_{3}+k\right) /\left(r_{3} \beta_{3}-1\right)}+\frac{c_{0}}{c_{2 m}}\left(\sum_{i=1}^{3}\left|y_{i}\right|\right) \operatorname{sgn}\left(\widetilde{w}_{3}\right)\right), \\
\tau_{2}=-\frac{1}{2}\left(l_{3} \widetilde{w}_{3}^{\left(r_{3}+k\right) /\left(r_{3} \beta_{3}-1\right)}+\frac{c_{0}}{c_{2 m}}\left(\sum_{i=1}^{3}\left|y_{i}\right|\right) \operatorname{sgn}\left(\widetilde{w}_{3}\right)\right. \\
\left.-k_{1} \operatorname{sgn}\left(\omega-c_{0}\right)\left|\omega-c_{0}\right|^{\alpha_{1}}\right),
\end{gathered}
$$

where

$$
\begin{gathered}
\widetilde{w}_{1}=y_{1}^{1+k}, \quad \widetilde{w}_{i}\left(y_{1}, \ldots, y_{i}\right)=y_{i}^{\beta_{i-1}}-\widetilde{v}_{i-1}^{\beta_{i-1}}, \\
\widetilde{v}_{i-1}=-l_{i-1} \widetilde{w}_{i-1}^{\left(r_{i-1}+k\right) /\left(r_{i-1} \beta_{i-2}\right)}, \quad i=2,3, \\
y_{1}=x \sin \theta-y \cos \theta, \quad y_{2}=c_{0}(x \cos \theta+y \sin \theta), \\
y_{3}=c_{0} v-c_{0}^{2}(x \sin \theta-y \cos \theta) .
\end{gathered}
$$

Unitl $y_{i}=0, i=1,2,3$, then go to Step $3^{\prime}$.

Step $3^{\prime}$. Let

$$
\tau_{1}=-\frac{1}{2} l_{2} \bar{w}_{2}^{\left(r_{2}+k\right) /\left(r_{2} \beta_{2}-1\right)}, \quad \tau_{2}=\frac{1}{2} l_{2} \bar{w}_{2}^{\left(r_{2}+k\right) /\left(r_{2} \beta_{2}-1\right)},
$$

where

$$
\begin{gathered}
\bar{w}_{1}=\theta^{1+k}, \quad \bar{w}_{2}(\theta, \omega)=\omega^{\beta_{1}}-\bar{v}_{1}^{\beta_{1}}, \\
\bar{v}_{1}=-l_{1} \bar{w}_{1}^{\left(r_{1}+k\right) /\left(r_{1} \beta_{0}\right)} .
\end{gathered}
$$

Until $\theta=\omega=0$, stop.

Then system (1) can be stabilized to the origin equilibrium point in a finite time by the switching controller Step $1^{\prime}$-Step $3^{\prime}$.

Proof. According to (25), we have the following:

$$
\left(\begin{array}{l}
x \\
y \\
\theta \\
v \\
\omega
\end{array}\right)=B\left(\begin{array}{l}
x_{1} \\
x_{2} \\
x_{3} \\
x_{4} \\
x_{5}
\end{array}\right),
$$

where

$$
B=\left(\begin{array}{ccccc}
0 & \cos x_{1} & \sin x_{1} & 0 & 0 \\
0 & \sin x_{1} & -\cos x_{1} & 0 & 0 \\
1 & 0 & 0 & 0 & 0 \\
0 & 0 & 0 & 0 & 1 \\
0 & 0 & 0 & 1 & 0
\end{array}\right)
$$

Because the determinant $\operatorname{det}(B)=1 \neq 0$, then it is obvious to see that $(x, y, \theta, v, \omega) \rightarrow 0$ is equivalent to $\left(x_{1}, x_{2}, x_{3}\right.$, $\left.x_{4}, x_{5}\right) \rightarrow 0$.

On the other hand, the switching controller $\left(u_{1}, u_{2}\right)$ in Theorem 4 can be used to stabilize the states $\left(x_{1}, x_{2}, x_{3}\right.$, $x_{4}, x_{5}$ ) of system (4) in a finite time; hence, the control task is changed to find the relation between the original controller $\left(\tau_{1}, \tau_{2}\right)$ of system $(1)$ and the controller $\left(u_{1}, u_{2}\right)$ of system $(4)$.

Comparing system (4) with system (1), we have the following:

$$
\begin{array}{cc}
\tau_{1}=\frac{u_{2}+u_{1}}{2}, & \tau_{2}=\frac{u_{2}-u_{1}}{2}, \\
c_{1}=\frac{R}{r I}, & c_{2}=\frac{1}{r m},
\end{array}
$$

from which, and by using the switching controller of Step 1Step 3 in Theorem 4, we can solve the corresponding $\left(\tau_{1}, \tau_{2}\right)$ and thus the conclusion can be obtained in Theorem 6 .

This completes the proof of the theorem.

\section{Simulations}

In this section, the discontinuous switching controller proposed in theorems above is used to show how to stabilize the state $\left(x_{1}, x_{2}, x_{3}, x_{4}, x_{5}\right)$ of (4) and the state $(x, y, \theta, v, \omega)$ of (1) to the zero equilibrium point in a finite time. We will demonstrate the effectiveness of our methods by a numerical example.

In the following simulation, we assume that $r_{m}=0.5$, $r_{M}=2.0, R_{m}=1.5, R_{M}=3.5, m_{m}=0.6, m_{M}=1.8, I_{m}=1.2$, $I_{M}=3.0$. The initial condition $(x(0), y(0), \theta(0), v(0), \omega(0))$ of system (1) is $(-0.2,-1.0,0.8,0.5,1.5)$. From (3) and (5), the initial value $\left(x_{1}(0), x_{2}(0), x_{3}(0), x_{4}(0), x_{5}(0)\right)$ of system (4) is $(0.8,-0.86,-0.84,1.5,0.5)$, and $c_{1 m}=0.25, c_{1 M}=5.83$, $c_{2 m}=0.28, c_{2 M}=3.33$. Given $c_{0}=1.0$, the design parameters are taken as follows: $k_{1}=2.5, \alpha_{1}=0.6, k=p / q-1=$ $7 / 9-1=-2 / 9, r_{1}=1, r_{2}=7 / 9, r_{3}=5 / 9, \beta_{0}=7 / 9, \beta_{1}=9 / 7$, $\beta_{2}=11 / 5, l_{1}=1.2, l_{2}=1.5, l_{3}=2.8$. The settling time in every step is given in advance; $T_{1}=5 \mathrm{~s}, T_{2}=20 \mathrm{~s}, T_{3}=30 \mathrm{~s}$.

Figures 2-5 show some simulation results with Matlab. From Figures 2 and 3, it can be seen that all the state variables of the closed system (4) are driven to the origin equilibrium point in a given settling time $T_{3}=30 \mathrm{~s}$. Observing Figure 2, in time interval $0 \sim 5 \mathrm{~s}$, the first step control task is completed, that is, $x_{4}=c_{0}=1$ as $t \geq 5 \mathrm{~s}$. Next, from Figure 3 , it is clear that $\left(x_{3}, x_{2}, x_{5}\right)$ can be stabilized to zero by the controller $u_{2}$ in Step 2 as $t \geq 20 \mathrm{~s}$ and remain unchanged. Finally, the controller $u_{1}$ in Step 3 drives $\left(x_{1}, x_{4}\right)$ to zero in the settling time $t \leq T_{3}$. 


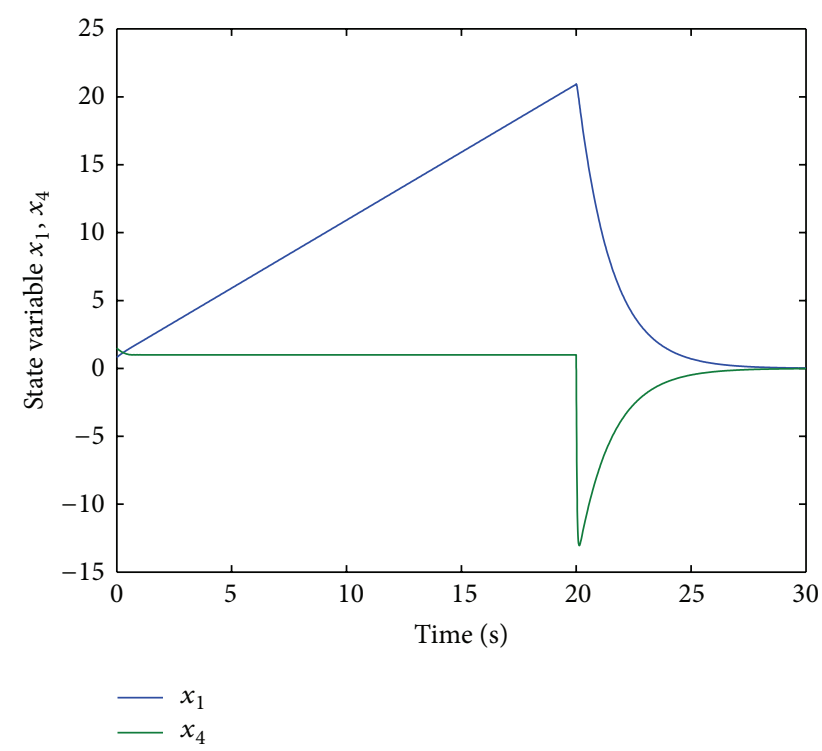

Figure 2: State variables $x_{1}, x_{4}$.

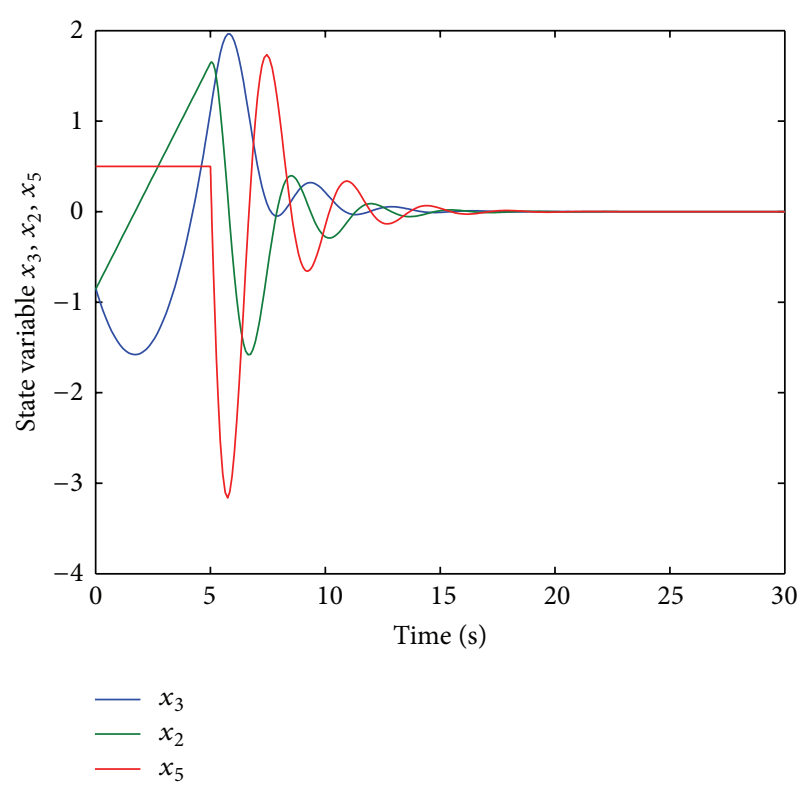

FIgURE 3: State variables $x_{3}, x_{2}$, and $x_{5}$.

Figures 4 and 5 show the finite-time convergence of the state variables $(x, y, \theta, v, \omega)$ of the original robot system (1) exactly as the state of the transformed system.

\section{Conclusion}

The robust finite-time stabilization problem is discussed in this paper for a class of uncertain dynamic nonholonomic wheeled mobile robot. The contributions of this paper include having applied finite-time control technique and a new switching design method such that all the states can be stabilized to the zero point by the proposed discontinuous

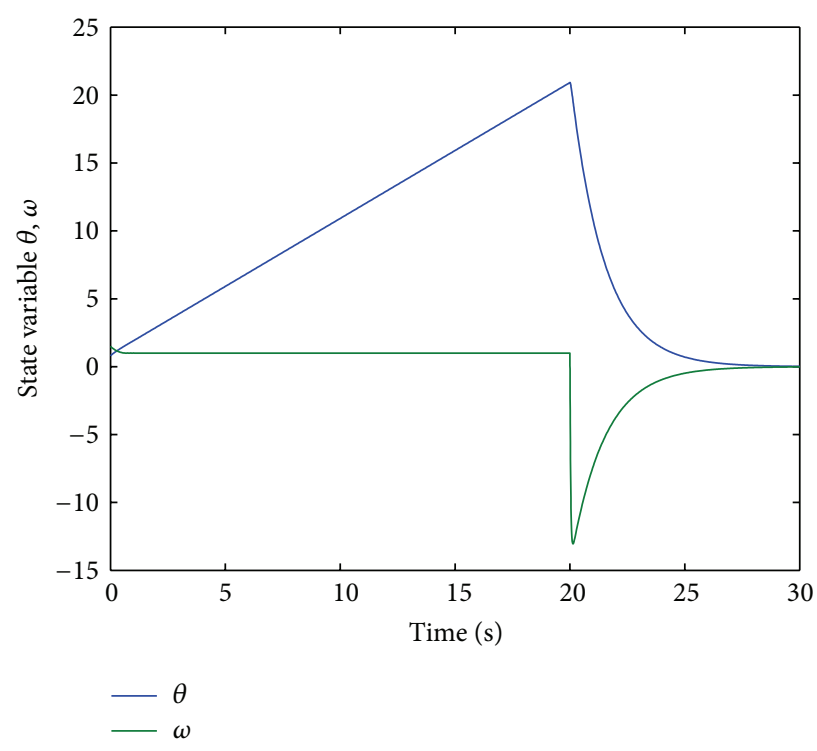

Figure 4: Original state variables $\theta, \omega$.

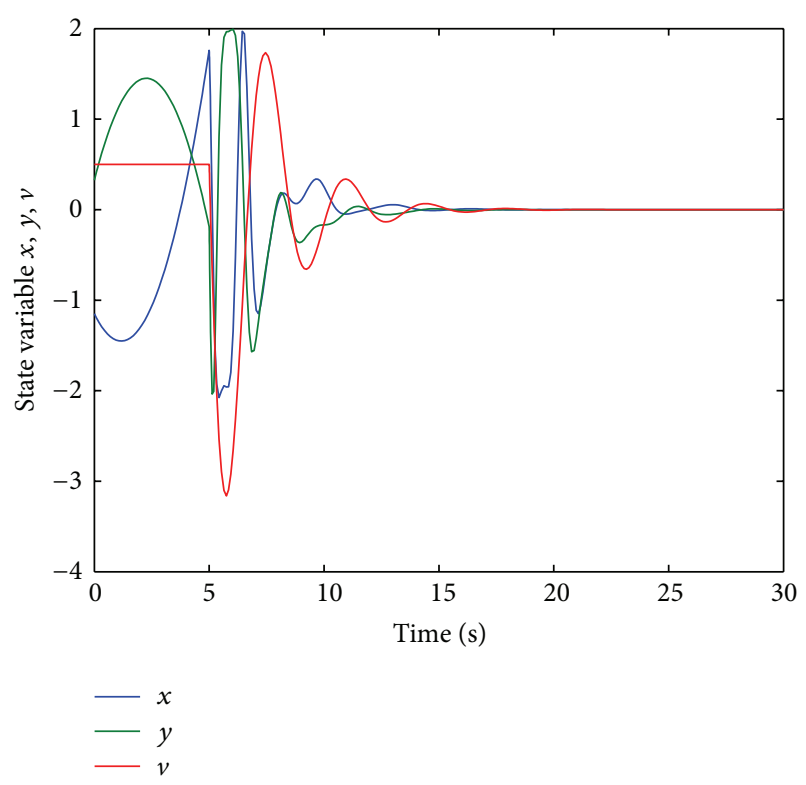

Figure 5: Original state variables $x, y$, and $v$.

controller. And we will work on extending the results to consider the corresponding trajectory tracking control problem in the coming time.

\section{Acknowledgments}

This paper was supported by the Natural Science Foundation of China (61304004, 61374040, and 11372097), China Postdoctoral Science Foundation (2013M531263), the Scientific Innovation Program of Shanghai Education Committee (13ZZ115), the National Basic Research Program of China (973 Project, 2010CB832702), the National Science Funds 
for Distinguished Young Scholars (11125208), the 111 Project (B12032), the R\&D Special Fund for Public Welfare Industry (Hydrodynamics Project, 201101014), and the the Natural Science Foundation of Hebei Province (A2014106035).

\section{References}

[1] R. W. Brockett, "Asymptotic stability and feedback stabilization," in Differential Geometric Control Theory, R. W. Brockett, R. S. Millman, and H. J. Sussmann, Eds., pp. 181-191, Birkhäauser, Boston, Mass, USA, 1983.

[2] R. M. Murray and S. S. Sastry, "Nonholonomic motion planning: steering using sinusoids," IEEE Transactions on Automatic Control, vol. 38, no. 5, pp. 700-716, 1993.

[3] Y.-P. Tian and S. Li, "Exponential stabilization of nonholonomic dynamic systems by smooth time-varying control," Automatica, vol. 38, no. 8, pp. 1139-1146, 2002.

[4] A. Teel, R. Murry, and G. Walsh, "Nonholonomic control systems: From steering to stabilization with sinusoids," in Proceedings of the IEEE Conference on Decision Control, pp. 1603-1609, 1992.

[5] A. Astolfi, "Discontinuous control of nonholonomic systems," Systems \& Control Letters, vol. 27, no. 1, pp. 37-45, 1996.

[6] H. Chen, J. Zhang, B. Chen et al., "Global practical stabilization for non-holonomic mobile robots with uncalibrated visual parameters by using a switching controller," IMA Journal of Mathematical Control and Information, 2013.

[7] A. M. Bloch and S. Drakunov, "Stabilization of a nonholonomic systems via sliding modes," in Proceedings of the IEEE Conference on Decision Control, pp. 2961-2963, 1995.

[8] C. Canudas de Wit and O. J. Sørdalen, "Exponential stabilization of mobile robots with nonholonomic constraints," IEEE Transactions on Automatic Control, vol. 37, no. 11, pp. 1791-1797, 1992.

[9] O. J. Sørdalen and O. Egeland, "Exponential stabilization of nonholonomic chained systems," IEEE Transactions on Automatic Control, vol. 40, no. 1, pp. 35-49, 1995.

[10] H. Chen, M. M. Ma, H. Wang, Z. Y. Liu, and Z. X. Cai, "Moving horizon $H_{\infty}$ tracking control of wheeled mobile robots with actuator saturation," IEEE Transactions on Control Systems Technology, vol. 17, no. 2, pp. 449-457, 2009.

[11] H. Chen, C. Wang, L. yang, and D. Zhang, "Semiglobal stabilization for nonholonomic mobile robots based on dynamic feedback with inputs saturation," Journal of Dynamic Systems, Measurement, and Control, vol. 134, no. 4, pp. 041006.1041006.8, 2012.

[12] H. Chen, C. Wang, B. Zhang, and D. Zhang, "Saturated tracking control for nonholonomic mobile robots with dynamic feedback," Transactions of the Institute of Measurement and Control, vol. 35, no. 2, pp. 105-116, 2013.

[13] B. L. Ma and S. K. Tso, "Robust discontinuous exponential regulation of dynamic nonholonomic wheeled mobile robots with parameter uncertainties," International Journal of Robust and Nonlinear Control, vol. 18, no. 9, pp. 960-974, 2008.

[14] Y. Hong, "Finite-time stabilization and stabilizability of a class of controllable systems," Systems \& Control Letters, vol. 46, no. 4, pp. 231-236, 2002.

[15] S. P. Bhat and D. S. Bernstein, "Continuous finite-time stabilization of the translational and rotational double integrators," IEEE Transactions on Automatic Control, vol. 43, no. 5, pp. 678-682, 1998.
[16] V. T. Haimo, "Finite time controllers," SIAM Journal on Control and Optimization, vol. 24, no. 4, pp. 760-770, 1986.

[17] Y. Q. Wu, B. Wang, and G. D. Zong, "Finite-time tracking controller design for nonholonomic systems with extended chained form," IEEE Transactions on Circuits and Systems II, vol. 52, no. 11, pp. 798-802, 2005.

[18] Y. Hong, J. Wang, and Z. Xi, "Stabilization of uncertain chained form systems within finite settling time," IEEE Transactions on Automatic Control, vol. 50, no. 9, pp. 1379-1384, 2005.

[19] S. Li and Y.-P. Tian, "Finite-time stability of cascaded timevarying systems," International Journal of Control, vol. 80, no. 4, pp. 646-657, 2007.

[20] Z. Wang, S. Li, and S. Fei, "Finite-time tracking control of a nonholonomic mobile robot," Asian Journal of Control, vol. 11, no. 3, pp. 344-357, 2009.

[21] M. Ou, H. Du, and S. Li, "Finite-time tracking control of multiple nonholonomic mobile robots," Journal of the Franklin Institute, vol. 349, no. 9, pp. 2834-2860, 2012. 


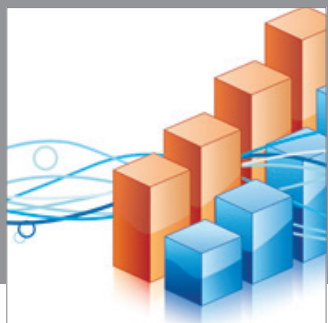

Advances in

Operations Research

mansans

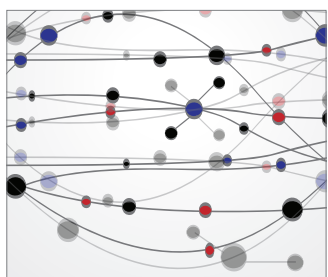

The Scientific World Journal
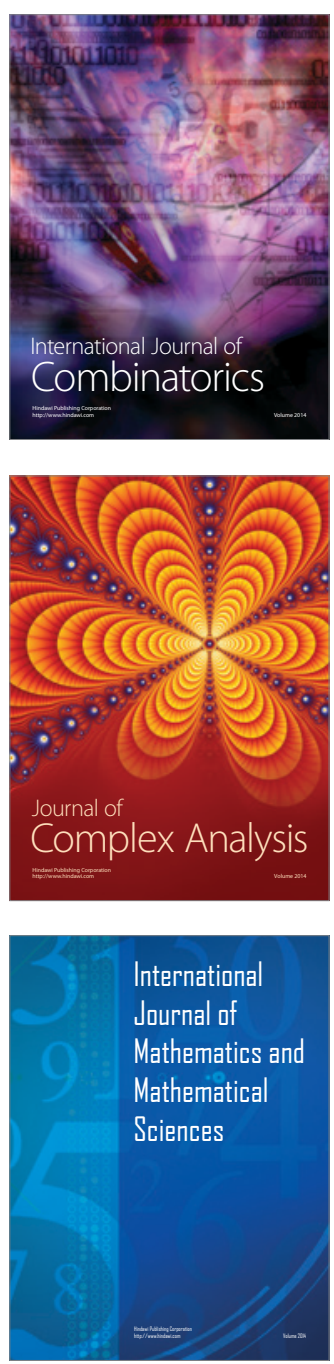
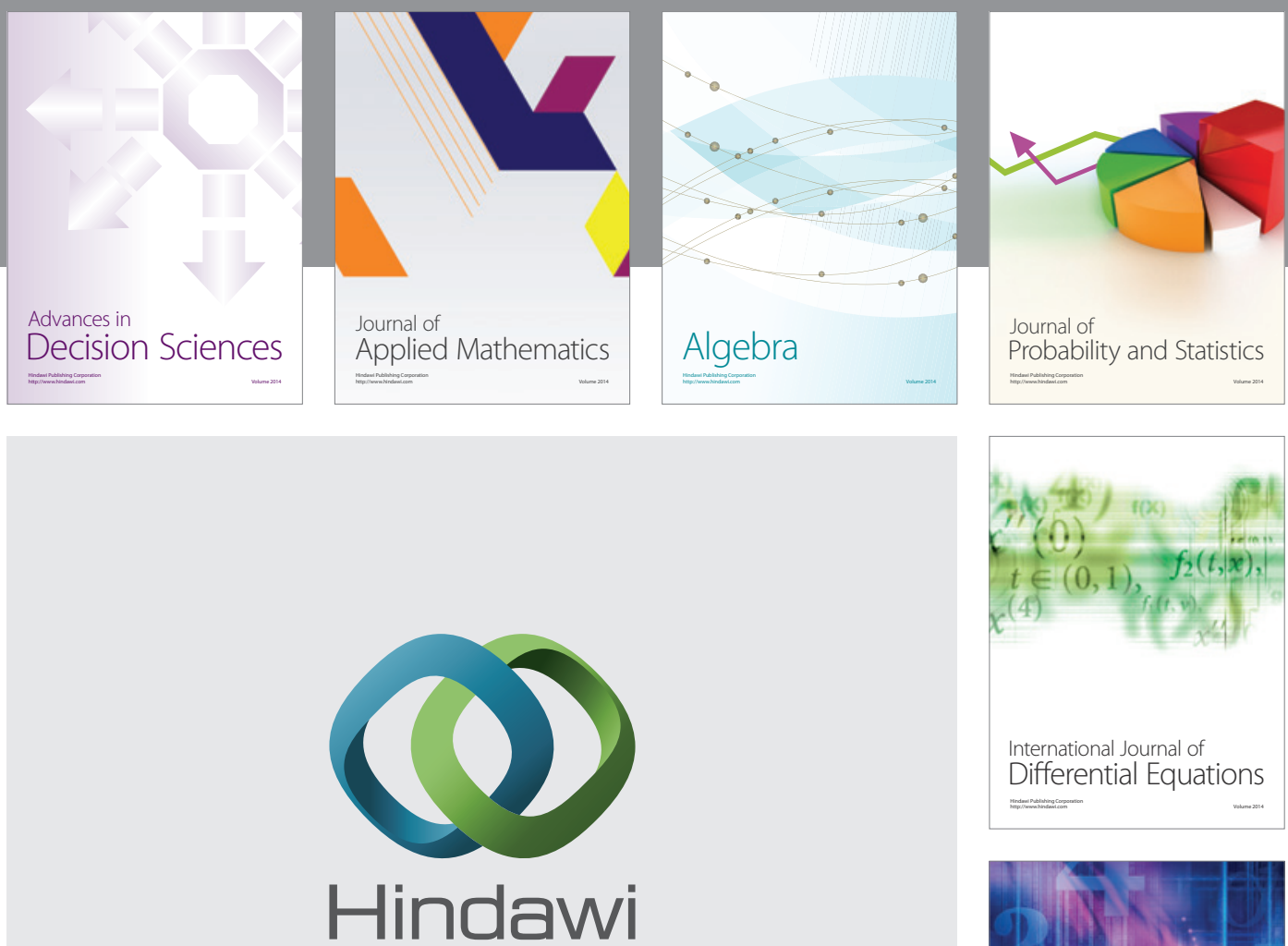

Submit your manuscripts at http://www.hindawi.com
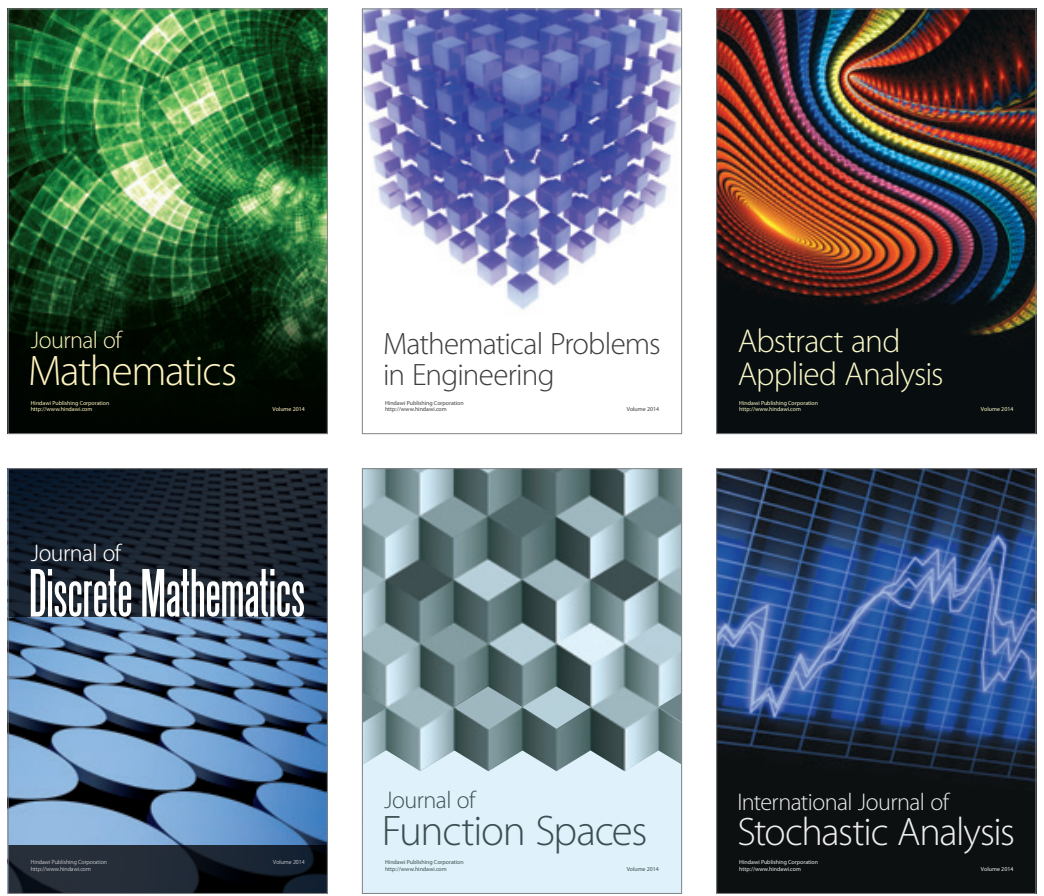

Journal of

Function Spaces

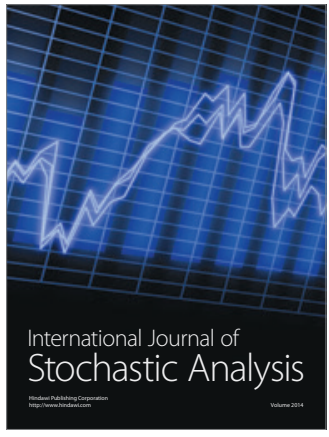

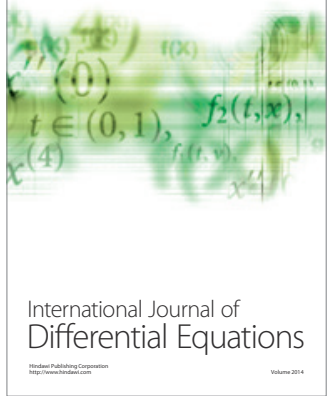
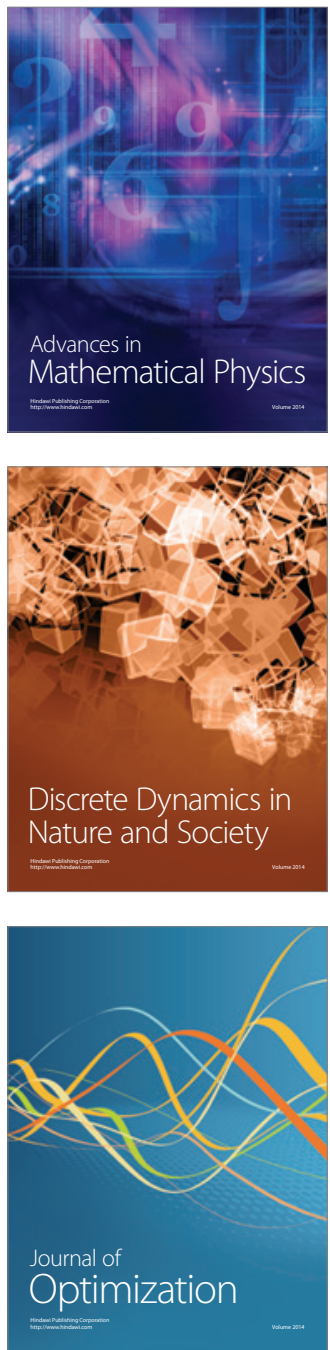